\title{
A NOTE ON EXTREMALLY DISCONNECTED SPACES
}

\author{
TAKASHI NOIRI
}

\begin{abstract}
A topological space $X$ is said to be locally $S$-closed if each point of $X$ has an open neighborhood which is an $S$-closed subspace of $X$. In this note it is shown that every locally $S$-closed weakly Hausdorff (or almost-regular) space is extremally disconnected.
\end{abstract}

1. Introduction. In 1976, T. Thompson [11] introduced the concept of $S$-closed spaces. Recently, the following sufficient conditions for an $S$-closed space to be extremally disconnected have been obtained.

Theorem A (HeRRMAnN [3]). An S-closed weakly Hausdorff space is extremally disconnected.

TheOREM B (HeRRMANN [3]). An S-closed almost-regular space is extremally disconnected.

TheOREM C (CAMERon [1]). A maximal S-closed space is extremally disconnected.

In [7], the present author introduced the concept of locally $S$-closed spaces which is strictly weaker than that of $S$-closed spaces. The purpose of the present note is to show that the condition " $S$-closed" in the theorems above stated can be replaced by "locally $S$-closed".

2. Preliminaries. Let $(X, \tau)$ be a topological space and $S$ a subset of $X$. The closure of $S$ and the interior of $S$ in $(X, \tau)$ are denoted by $\mathrm{Cl}_{X}(S)$ and $\operatorname{Int}_{X}(S)$, respectively. A subset $S$ of $X$ is said to be regular open (regular closed) if $\operatorname{Int}_{X}\left(\mathrm{Cl}_{X}(S)\right)=S$ (resp. $\left.\mathrm{Cl}_{X}\left(\operatorname{Int}_{X}(S)\right)=S\right)$. A topological space $(X, \tau)$ is said to be extremally disconnected if $\mathrm{Cl}_{X}(U) \in \tau$ for every $U \in \tau$. A subset $S$ of $(X, \tau)$ is said to be semiopen [4] if there exists $U \in \tau$ such that $U \subset S \subset \mathrm{Cl}_{X}(U)$. The family of all semiopen sets in $(X, \tau)$ is denoted by $\operatorname{SO}(X, \tau)$.

Definition 2.1. A topological space $(X, \tau)$ is said to be $S$-closed [11] if for every semiopen cover $\left\{U_{\alpha} \mid \alpha \in \nabla\right\}$ of $X$ there exists a finite subfamily $\nabla_{0}$ of $\nabla$ such that $X=\cup\left\{\mathrm{Cl}_{X}\left(U_{\alpha}\right) \mid \alpha \in \nabla_{0}\right\}$.

A subset $S$ of $(X, \tau)$ is said to be $S$-closed if it is $S$-closed as the subspace of $(X, \tau)$. A subset $S$ of $(X, \tau)$ is said to be $S$-closed relative to $\tau$ [6] if for every cover $\left\{U_{\alpha} \mid \alpha \in \nabla\right\}$ of $S$ by semiopen sets of $(X, \tau)$ there exists a finite subfamily $\nabla_{0}$ of $\nabla$ such that $S \subset \cup\left\{\mathrm{Cl}_{X}\left(U_{\alpha}\right) \mid \alpha \in \nabla_{0}\right\}$.

Received by the editors November 9, 1978 and, in revised form, March 19, 1979.

AMS (MOS) subject classifications (1970). Primary 54D20; Secondary 54G05.

$K e y$ words and phrases. $S$-closed, locally $S$-closed, extremally disconnected. 
Definition 2.2. A topological space $(X, \tau)$ is said to be locally $S$-closed [7] if each point of $X$ has an open neighborhood which is an $S$-closed subspace of $(X, \tau)$.

Every $S$-closed space is locally $S$-closed. However, the converse is not true in general because an infinite discrete space is locally $S$-closed but not $S$-closed. The following lemmas shown in [7] will be used in the sequel.

LemMA 2.3. Let $A$ and $B$ be subsets of a topological space $(X, \tau)$. If $A$ is $S$-closed relative to $\tau$ and $B$ is regular open, then $A \cap B$ is $S$-closed relative to $\tau$.

LEMMA 2.4. For a topological space $(X, \tau)$, the following are equivalent.

(1) $(X, \tau)$ is locally $S$-closed.

(2) For each $x \in X$, there exists $U \in \tau$ such that $x \in U$ and $U$ is $S$-closed relative to $\tau$.

(3) For each $x \in X$, there exists $U \in \tau$ such that $x \in U$ and $\operatorname{Int}_{X}\left(\mathrm{Cl}_{X}(U)\right)$ is $S$-closed relative to $\tau$.

3. The results.

Definition 3.1. A topological space $(X, \tau)$ is said to be weakly Hausdorff [10] if each point of $X$ is an intersection of regular closed sets of $(X, \tau)$.

TheOREM 3.2. If a topological space $(X, \tau)$ is locally $S$-closed and weakly Hausdorff, then it is extremally disconnected.

Proof. Assume that $(X, \tau)$ is not extremally disconnected. Then, there exists a regular open set $G$ of $(X, \tau)$ such that $\mathrm{Cl}_{X}(G)-G \neq \varnothing$ and $X-\mathrm{Cl}_{X}(G) \neq \varnothing$. Let $x \in \mathrm{Cl}_{X}(G)-G$. By Lemma 2.4, there exists an open neighborhood $V$ of $x$ such that $V$ is $S$-closed relative to $\tau$. Put $A=G \cap V$, then by Lemma 2.3, $A$ is $S$-closed relative to $\tau$. Since $(X, \tau)$ is weakly Hausdorff and $x \notin A$, for each $a \in A$ there exists a regular closed set $F(a)$ such that $x \notin F(a)$ and $a \in F(a)$. Since $F(a) \in$ $\mathrm{SO}(X, \tau)$, there exists a finite subset $A_{0}$ of $A$ such that $A \subset \cup\left\{F(a) \mid a \in A_{0}\right\}$. We have $A \subset V \cap \mathrm{Cl}_{X}(G) \subset \mathrm{Cl}_{X}(V \cap G)=\mathrm{Cl}_{X}(A)$. Therefore, $x \in \mathrm{Cl}_{X}(A) \subset$ $\cup\left\{F(a) \mid a \in A_{0}\right\}$. On the other hand, $x \notin F(a)$ for any $a \in A_{0}$ and hence $x \notin$ $\cup\left\{F(a) \mid a \in A_{0}\right\}$. This contradiction implies that $(X, \tau)$ is extremally disconnected.

Corollary 3.3. A weakly Hausdorff space $(X, \tau)$ is $S$-closed if and only if it is locally $\mathrm{S}$-closed and quasi $\mathrm{H}$-closed.

DefinItion 3.4. A topological space $(X, \tau)$ is said to be almost-regular [9] if for each $x \in X$ and each regular closed set $F$ not containing $x$ there exist disjoint open sets $U$ and $V$ of $(X, \tau)$ such that $x \in U$ and $F \subset V$.

THEOREM 3.5. If a topological space $(X, \tau)$ is locally $S$-closed and almost-regular, then it is extremally disconnected.

Proof. Assume that $(X, \tau)$ is not extremally disconnected. Then there exists a regular open set $G$ of $(X, \tau)$ such that $\mathrm{Cl}_{X}(G)-G \neq \varnothing$ and $X-\mathrm{Cl}_{X}(G) \neq \varnothing$. Let $x \in \mathrm{Cl}_{X}(G)-G$. Then, by Lemma 2.4 , there exists a regular open neighborhood $O$ of $x$ which is $S$-closed relative to $\tau$. Put $A=O \cap G$, then by Theorem 1.2 of [6] 
and Lemma 2.3, $A$ is an $S$-closed subspace of $(X, \tau)$. Let $\mathfrak{B}(x)$ be the family of all neighborhoods at $x$ and $\mathscr{F}=\{V \cap A \mid V \in \mathfrak{B}(x)\}$. Then, $\mathscr{F}$ is a filter base on $A$ and hence, by Theorem 2 of [11], $\mathscr{F} s$-accumulates to some point $a \in A$. Since $(X, \tau)$ is almost-regular and $A$ is regular open, there exists $U \in \tau$ such that $a \in U \subset \mathrm{Cl}_{X}(U) \subset A$ [9, Theorem 2.2]. Since $x \notin A,\left(X-\mathrm{Cl}_{X}(U)\right) \cap A \in \mathcal{F}$ and $a \in U \in \mathrm{SO}(A)$. Moreover, we have $\left[\left(X-\mathrm{Cl}_{X}(U)\right) \cap A\right] \cap \mathrm{Cl}_{A}(U)=\varnothing$ which contradicts that $\mathscr{F} s$-accumulates to $a \in A$. This shows that $(X, \tau)$ is extremally disconnected.

COROLlary 3.6. An almost-regular space $(X, \tau)$ is $S$-closed if and only if it is locally $\mathrm{S}$-closed and quasi $\mathrm{H}$-closed.

Definition 3.7. A locally $S$-closed space $(X, \tau)$ is said to be maximal locally $S$-closed if $\tau=\theta$ whenever a topological space $(X, \theta)$ is locally $S$-closed and $\theta$ is stronger than $\tau$.

A function $f:(X, \tau) \rightarrow(Y, \theta)$ is said to be irresolute [2] if $f^{-1}(V) \in \operatorname{SO}(X, \tau)$ for every $V \in \operatorname{SO}(Y, \theta)$.

LeMma 3.8. Let $f:(X, \tau) \rightarrow(Y, \theta)$ be an irresolute function. If $G \in \tau$ and $G$ is $S$-closed in $(X, \tau)$, then $f(G)$ is $S$-closed in $(Y, \theta)$.

Proof. Let $f_{G}: G \rightarrow f(G)$ be a function defined by $f_{G}(x)=f(x)$ for every $x \in G$, where $G$ (resp. $f(G))$ is the subspace of $(X, \tau)$ (resp. $(Y, \theta)$ ). We shall show that $f_{G}$ is irresolute. For any $V_{0} \in \operatorname{SO}(f(G))$, there exists $V \in \operatorname{SO}(Y, \theta)$ such that $V_{0}=V$ $\cap f(G)$ [8, Theorem 3.2]. Since $f$ is irresolute and $G \in \tau, f^{-1}(V) \cap G \in \operatorname{SO}(X, \tau)$ and hence, by Theorem 1 of [5], $f_{G}^{-1}\left(V_{0}\right)=f^{-1}(V) \cap G \in \operatorname{SO}(G)$. This shows that $f_{G}$ is irresolute. Since $G$ is $S$-closed, it follows from Theorem 3.5 of [12] that $f_{G}(G)=f(G)$ is $S$-closed.

TheOREM 3.9. If a topological space $(X, \tau)$ is maximal locally $S$-closed, then it is extremally disconnected.

Proof. Assume that $(X, \tau)$ is not extremally disconnected. Then, there exists a regular closed set $B$ of $(X, \tau)$ such that $B \notin \tau$. Put $\tau(B)=\{U \cup(V \cap B) \mid$ $U, V \in \tau\}$, then $\tau(B)$ is a topology on $X$ which is strictly stronger than $\tau$. We shall show that $(X, \tau(B))$ is locally $S$-closed. Let $i_{X}:(X, \tau) \rightarrow(X, \tau(B))$ be the identity function. Then, it is obvious that $i_{X}$ is open. For each $U \cup(V \cap B) \in \tau(B)$, $V \cap B \in \operatorname{SO}(X, \tau)$ and hence $U \cup(V \cap B) \in \operatorname{SO}(X, \tau)$. This shows that $i_{X}$ is semicontinuous. Therefore, $i_{X}$ is irresolute [5, Theorem 7]. Since $(X, \tau)$ is locally $S$-closed, for each $x \in X$ there exists an open neighborhood $U$ of $x$ in $(X, \tau)$ such that $U$ is $S$-closed. By Lemma $3.8, i_{X}(U)$ is $S$-closed in $(X, \tau(B))$. Moreover, $i_{X}(U)$ is an open neighborhood of $x$ in $(X, \tau(B))$ because $i_{X}$ is open. This shows that $(X, \tau(B))$ is locally $S$-closed. This contradicts that $(X, \tau)$ is maximal locally $S$ closed. Therefore, $(X, \tau)$ is extremally disconnected.

The author is sincerely grateful to the referee for many valuable suggestions. 


\section{REFERENCES}

1. D. E. Cameron, Properties of S-closed spaces, Proc. Oklahoma Toplogy Conf., Oklahoma, 1978.

2. S. G. Crossley and S. K. Hildebrand, Semi-topological properties, Fund. Math. 74 (1972), 233-254.

3. R. A. Herrmann, $A$ nonstandard approach to $S$-closed spaces (RC-convergence), Notices Amer. Math. Soc. 25 (1978), A-364.

4. N. Levine, Semi-open sets and semi-continuity in topological spaces, Amer. Math. Monthly 70 (1963), 36-41.

5. T. Noiri, On semi-continuous mappings, Atti Accad. Naz. Lincei Rend. C. Sci. Fis. Mat. Natur. (8) 54 (1973), 210-214.

6. __ On S-closed spaces, Ann. Soc. Sci. Bruxelles Sér. I. 91 (1977), 189-194.

7. (1978), 157-162.

8. V. Pipitone and G. Russo, Spazi semiconnessi e spazi semiaperti, Rend. Circ. Mat. Palermo (2) 24 (1975), 273-285.

9. M. K. Singal and S. P. Arya, On almost-regular spaces, Glasnik Mat. Ser. III (4) 24 (1969), 89-99.

10. T. Soundararajan, Weakly Hausdorff spaces and the cardinality of topological spaces, General Topology and its Relations to Modern Analysis and Algebra. III, Proc. Conf. Kampur, 1968, Academia, Prague, 1971, pp. 301-306.

11. T. Thompson, S-closed spaces, Proc. Amer. Math. Soc. 60 (1976), 335-338.

12. Semicontinuous and irresolute images of S-closed spaces, Proc. Amer. Math. Soc. 66 (1977), 359-362.

Yatsushiro College of Technology, Yatsushiro, Kumamoto, 866 JAPAN 